\title{
Mechanical analysis of flexible integrated energy storage devices under bending by the finite element method
}

\author{
Zishun Yuan ${ }^{1,2}$, Minjie Yao ${ }^{1}$, Nannan Zhang ${ }^{1}$, Shuai Wang ${ }^{1}$, Xianhong Rui ${ }^{2}$, Qichun Zhang $^{3}$ and \\ Zhiqiang $\mathrm{Niu}^{1 *}$
}

\begin{abstract}
Although a great deal of studies focus on the design of flexible energy storage devices (ESDs), their mechanical behaviors under bending states are still not sufficiently investigated, and the understanding of the corresponding structural conversion therefore still lags behind. Here, we systematically and thoroughly investigated the mechanical behaviors of flexible all-in-one ESDs under bending deformation by the finite element method. The influences of thicknesses, Young's moduli and Poisson's ratios of electrodes and electrolyte were taken into account. Visualized and quantified results including displacement, strain energy, von Mises stress, and tensile, compressive, and interfacial shear stress are demonstrated and analyzed. Based on these results, significant conclusions are drawn for the design of flexible integrated ESDs with robust mechanical properties. This work will provide guidance for the design of ESDs with high flexibility.
\end{abstract}

Keywords: energy storage devices, all-in-one, flexible, finite element method, mechanical analysis

\section{INTRODUCTION}

Flexible electronics are increasingly applied in a diversity of areas, such as foldable communication devices, wearable electronic devices, and surgical and diagnostic implements. As one of the most important parts in the flexible electronics, flexible energy storage devices (ESDs) are recently drawing more and more attention [1-9]. They are required to not only have great electrochemical properties, but also possess desirable flexibility to tolerate high strain induced by external mechanical deformation such as bending, stretching, and twisting [10-13]. Bending is one of the most common mechanical deformations for flexible electrical devices in daily use. Therefore, it is of paramount significance to understand the mechanical properties and structural conversion of the ESDs under different bending levels, which are able to prevent the irreversible deformation under bending states.

Recently, flexible ESDs with various configurations have been developed [1-6,14-16]. Among these different configurations, all-in-one architecture, where all the components of the device are integrated together with continuous seamless connection, can display the ability to endure larger bending strains in comparison with the conventional sandwiched structure [17-20]. Therefore, such structure can achieve more stable electrochemical performance during and after bending deformation. However, heretofore, the analyses for this structure in terms of mechanical behaviors by experimental and analytical methods were not sufficiently quantified and unambiguous. More detailed and quantitative information, like how tensile, compressive, and shear stresses distribute on the whole flexible integrated ESDs during bending, has to be further understood, which will be beneficial to optimize the design of flexible integrated ESDs.

Finite element (FE) method, a numerical method based on the calculus of variations, is invariably used in the field of mechanics. The mechanical behaviors of various materials and structures can be predicted and analyzed by

\footnotetext{
${ }^{1}$ Key Laboratory of Advanced Energy Materials Chemistry (Ministry of Education), Renewable Energy Conversion and Storage Center, College of Chemistry, Nankai University, Tianjin 300071, China

${ }^{2}$ School of Materials and Energy, Guangdong University of Technology, Guangzhou 510006, China

${ }^{3}$ Department of Materials Science and Engineering, City University of Hong Kong, Kowloon 999077, Hong Kong, China

* Corresponding author (email: zqniu@nankai.edu.cn)
} 
the visualized and quantitative details from the FE modelling [21-29]. Here, we make a systematic analysis on the mechanical behaviors of flexible integrated ESDs at various bending states using the FE method. Such method can illustrate not only the strain energy (SE) at different bending states, but also various strains and stresses in the whole curved ESDs. Therefore, the dependence of mechanical properties of the ESDs on the Young's moduli and thicknesses of electrodes and electrolyte is investigated systematically. These findings will provide the guidance for the design of flexible integrated ESDs.

\section{RESULTS AND DISCUSSION}

\section{Establishment of symmetric integrated ESD models}

The flexible integrated ESDs are generally fabricated by using carbon-based materials as the frameworks of the electrodes, which play major roles in the mechanical properties of the electrodes [2]. Thus, it is feasible to make an integrated ESD whose electrodes possess similar mechanical properties by using similar frameworks for their two electrodes. In this work, in order to make the FE analysis easier to be comprehended, such construction can be assumed as symmetry in terms of structures and mechanical properties and the symmetric integrated ESDs were used as the representative structures for the FE modeling.

Fig. 1a illustrates a sketch of one typical all-in-one ESD at a bending state. In such sketch, the electrolyte acts as the separator, and diffuses into the porous electrodes, achieving an all-in-one integrated architecture. The yellow and blue parts represent electrolyte and electrodes, respectively, while the parts with the gradient color re- present the integrated interfaces formed by merging the electrolyte with the electrodes. The coordinate axis is presented to define the $x, y$, and $z$ directions for the global system. For the sake of accuracy and convenience in the following description, we defined direction 11 to be completely along the longitudinal edges of the ESD, even if they are curved, while defined direction 33 to be vertical to the top or bottom surface. Thus, direction 33 of different areas in the top or bottom surfaces is different when the ESD is curved. An infinitesimal representative cell (IRC) was selected from the center of the curved ESD, by which the theoretical analysis in terms of stress and strain distributions is demonstrated. The classical beam theory in bending from the material mechanics was used because it is easier to be comprehended and accurate for an IRC in a very small deflection (normally distance of its deflection is smaller than the thickness of the beam) [30]. From the side view of the cell, specifically, curve $A B$ is the neutral line and in the middle of the cross-section due to the symmetric structure. $\mathrm{O}$ is the center of curvature of the curve $\mathrm{AB}$ and $\theta$ is the angle to the curve. $M$ represents the bending moment of the representative cell. Curve CD is paralleled to the neutral line, with their distance referred to as $y$. When the curve $\mathrm{CD}$ is upon the neutral line, $y$ is considered to be positive and the strain along the $\mathrm{CD}$ is tensile, whereas the situations are opposite when the curve $\mathrm{CD}$ is beneath. The tensile or compressive strain and stress along the curve CD, referred to as $\varepsilon_{11}$ and $\sigma_{11}$, respectively, here are almost the same as $\varepsilon_{x}$ and $\sigma_{x}$ (for the IRC, direction 11 and direction $x$ are almost coincident), and therefore are expressed as follows:

$\varepsilon_{x}=\frac{y}{\rho}$
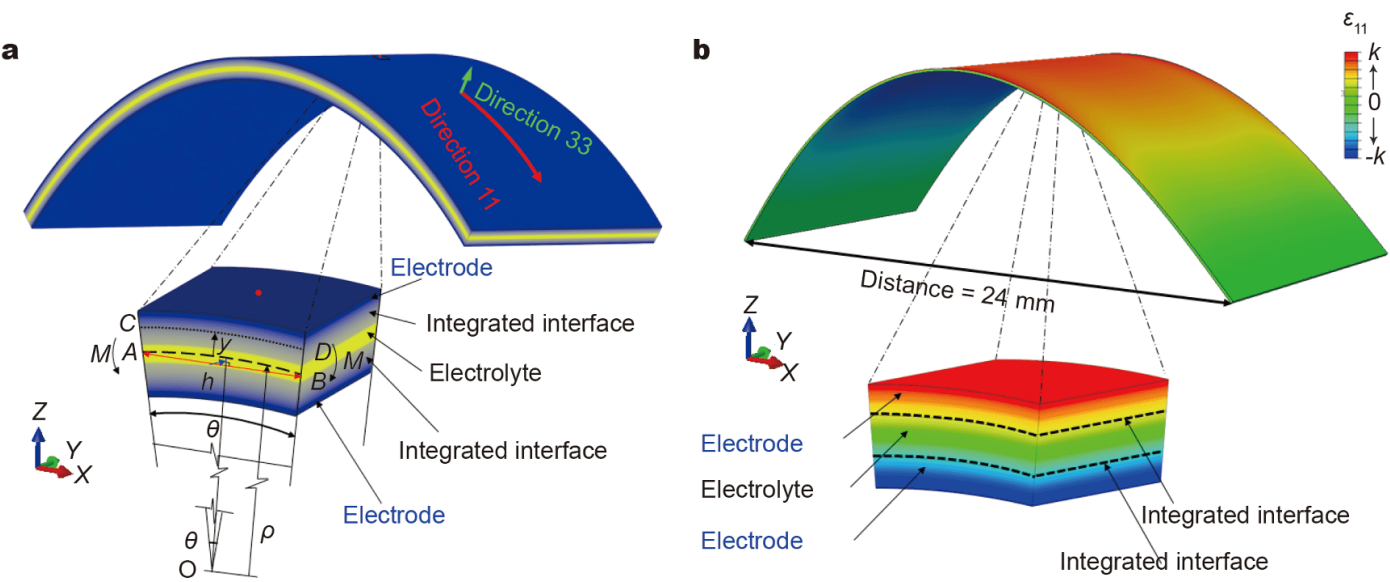

Figure 1 Development of the finite element (FE) model. (a) A sketch of the analytical model of the flexible integrated ESDs under bending. (b) The FE model under bending. 
$\sigma_{x}=E \times \varepsilon_{x}$,

where $\rho$ is the radius of curvature of the $\mathrm{AB}$, and $E$ is the Young's modulus of the material at the curve CD. $\rho$ can be calculated by the following equation:

$\rho=\frac{l^{2}}{8 h}+\frac{h}{2}$,

where $l$ is the distance of line $\mathrm{AB}$ and $h$ is the vertical distance from the middle point of the curve $A B$ to the line.

According to the above three equations (1-3), the Young's moduli and thicknesses of the electrodes and electrolyte are the main influential parameters to the stress and strain in the flexible ESDs, which can be investigated by $\mathrm{FE}$ modeling. A geometric model of the symmetric integrated ESD was developed, whose length, width, and thickness are 30,10 , and $0.1 \mathrm{~mm}$, respectively (Fig. S1). The thicknesses of the electrodes and electrolyte, referred to as $d_{\text {electrode }}$ and $d_{\text {electrolyte, }}$ are initially set to 0.025 and $0.05 \mathrm{~mm}$, respectively. Materials used for the electrode and electrolyte were considered to be homogeneous and isotropic. Their elastic moduli and Poisson ratios, denoted by $E_{\text {electrode }}, E_{\text {electrolyte, }}, v_{\text {electrode }}$, and $v_{\text {electrolyte, }}$, were initially set to $1000 \mathrm{MPa}, 100 \mathrm{MPa}, 0.3$, and 0.2 , respectively. The values of $E_{\text {electrode }}, E_{\text {electrolyte, }}, d_{\text {electrode }}$, and $d_{\text {electrolyte }}$ will be changed to investigate their influences. The definitions of the coordinate axis and direction 11 and 33 are identical to those in the analytical model. To make the model curved, we applied upward displacement $(0.5 \mathrm{~mm})$ along $z$ axis to the middle of the model, and then inward displacement along $x$ axis to the short edges on the bottom surface (Fig. S1). More details, including the setup of boundary conditions and the study of mesh convergence, are described in the Supplementary information (Figs S1, S2).

Fig. $1 \mathrm{~b}$ demonstrates $\varepsilon_{11}$ of the curved ESD model. The colors on the model represent the different scales and types of $\varepsilon_{11}$. The values upon the neutral plane are positive, which indicates the tensile strains, while beneath are negative, which indicates the compressive strains. It is shown that $\varepsilon_{11}$ reaches maximum in the middle of the model on both top and bottom surface, and gradually decreases towards the two ends. Moreover, since the curvature in the three-dimensional (3D) bent model is difficult to be measured with accuracy, the bending state of this model was defined using end-to-end distance $(D)$ between the edges of longitudinal ends, instead of using the bending curvature. The bending status of the curved ESD model in Fig. $1 \mathrm{~b}$ is corresponding to $D=24 \mathrm{~mm}$. According to the interface characteristic of the integrated structure, which is continuous and seamless, the interface between one electrode and the electrolyte (separator) in the integrated ESD model was assumed to be, for simplicity, infinitesimal in thickness, by which they are perfectly bonded. In order to validate the FE model, $\varepsilon_{11}$ at the central points of the bottom and top surfaces calculated by the FE method at all the bending states were compared with the analytical counterparts. The pertinent details are elucidated in the Supplementary information (Fig. S3). Their consistent results indicate that the developed FE model is theoretically reasonable.

\section{Influences of Young's modulus and thickness of the electrodes on SE and von Mises stress of the ESDs}

To investigate the influences of $d_{\text {electrode }}, E_{\text {electrode }}, d_{\text {electrolyte, }}$, and $E_{\text {electrolyte }}$ on SE and von Mises stress (or Mises stress) of the ESDs, four groups denoted by Groups 1, 2, 3, and 4 were developed, with one variable ( $d_{\text {electrode }}, E_{\text {electrode }}$, $d_{\text {electrolyte, or }} E_{\text {electrolyte}}$ ) in each group, respectively (Table 1). The values for each variable were selected from references, covering the range of almost all the Young's moduli and thicknesses of materials used in the corresponding components of the flexible ESDs [31-48]. The ratios of $d_{\text {electrode }}$ to $d_{\text {electrolyte }}$ and $E_{\text {electrode }}$ to $E_{\text {electrolyte }}$ were defined as $\mu$ and $\lambda$, respectively (Fig. 2a). SE and Mises stress are the main outputs selected for the quantified analysis. SE is the energy from elastic deformation, and is able to evaluate how flexible those ESD models are. Mises stress is a scalar value and the result of the Cauchy stress tensors, which can be used to predict where the yield, damage, or failure is easier to occur. In order to quantify the stress distribution (instead of presenting it only in different colors), the paths along the middle lines in direction 11 and direction $y$ on the top surface were defined to be Path T11 and Path TY, respectively, at one representative bending state $D=24 \mathrm{~mm}$ (Fig. 2b). The corresponding paths on the bottom surface were defined as Path B11 and Path BY.

From a general view, SE of each case in Groups 1 and 2 overall linearly increases as $D$ decreases from 30 to $10 \mathrm{~mm}$ (Fig. 2c, f). The results indicate that regarding each case in Groups 1 and 2, the increase of the bending extent requires more work from outside in proportion. The results can be more intuitionistic by displaying $\varepsilon_{11}$ of one model at different bending states (because $\varepsilon_{11}$ is the principal strain); the strains on both top and bottom surfaces intensify as $D$ decreases, especially at their peak regions (Fig. 2f). Concerning Mises stress of each model in Groups 1 and 2, the values along Path T11 and Path TY are overall symmetric about the axis $\mathrm{T} 11=0$ and $\mathrm{TY}=0$, 
Table 1 Four groups with one variable $\left(d_{\text {electrode }}, E_{\text {electrode }}, d_{\text {electrolyte}}\right.$, or $E_{\text {electrolyte })}$ in each

\begin{tabular}{|c|c|c|c|c|c|c|}
\hline & $d_{\text {electrode }}(\mathrm{mm})$ & $E_{\text {electrode }}(\mathrm{MPa})$ & $d_{\text {electrolyte }}(\mathrm{mm})$ & $E_{\text {electrolyte }}(\mathrm{MPa})$ & $\mu=d_{\text {electrode }} / d_{\text {electrolyte }}$ & $\lambda=E_{\text {electrode }} / E_{\text {electrolyte }}$ \\
\hline \multirow{5}{*}{ Group 1} & $0.005[31]$ & \multirow{5}{*}{1000} & \multirow{5}{*}{0.05} & \multirow{5}{*}{100} & 0.1 & \multirow{5}{*}{10} \\
\hline & $0.01[31]$ & & & & 0.2 & \\
\hline & $0.025[32]$ & & & & 0.5 & \\
\hline & $0.05[33]$ & & & & 1 & \\
\hline & $0.1[34]$ & & & & 2 & \\
\hline \multirow{11}{*}{ Group 2} & \multirow{11}{*}{0.025} & $10[35]$ & \multirow{11}{*}{0.05} & \multirow{11}{*}{100} & \multirow{11}{*}{0.5} & 0.1 \\
\hline & & $50[36]$ & & & & 0.5 \\
\hline & & $100[37]$ & & & & 1 \\
\hline & & $500[38]$ & & & & 5 \\
\hline & & $1000[39]$ & & & & 10 \\
\hline & & $2500[40]$ & & & & 25 \\
\hline & & $5000[41]$ & & & & 50 \\
\hline & & $10000[42]$ & & & & 100 \\
\hline & & $25000[43]$ & & & & 250 \\
\hline & & $50000[43]$ & & & & 500 \\
\hline & & $100000[44]$ & & & & 1000 \\
\hline \multirow{6}{*}{ Group 3} & \multirow{6}{*}{0.025} & \multirow{6}{*}{1000} & 0.01 & \multirow{6}{*}{100} & 2.5 & \multirow{6}{*}{10} \\
\hline & & & $0.025[45]$ & & 1 & \\
\hline & & & $0.05[33]$ & & 0.5 & \\
\hline & & & $0.1[34]$ & & 0.25 & \\
\hline & & & $0.15[33]$ & & 0.167 & \\
\hline & & & $0.2[33]$ & & 0.125 & \\
\hline \multirow{7}{*}{ Group 4} & \multirow{7}{*}{0.025} & \multirow{7}{*}{1000} & \multirow{7}{*}{0.05} & $10[46]$ & & 100 \\
\hline & & & & $50[37]$ & & 20 \\
\hline & & & & $100[37]$ & & 10 \\
\hline & & & & $500[47]$ & 0.5 & 2 \\
\hline & & & & $1000[47]$ & & 1 \\
\hline & & & & $2500[48]$ & & 0.4 \\
\hline & & & & $5000[47]$ & & 0.2 \\
\hline
\end{tabular}

respectively (Fig. 2d, e, g, h and Figs S4, S5). Along the Path T11, the stresses gradually decrease from the middle to the two ends (Fig. 2d, g). Along the Path TY, the stresses present almost flat from the middle to around $\pm 4 \mathrm{~mm}$, and increase to the maximum at the two ends, respectively (Fig. 2e, h). The situation along Path BY is partly different from that along the Path TY; the stresses gradually decrease from around $\pm 4 \mathrm{~mm}$ to the ends, though still keep overall constant between around -4 and $4 \mathrm{~mm}$ (Figs S6, S7). Specific to the influence of the thickness of electrodes, SE at any bending states increases as $\mu$ increases from 0.1 to 2 (Fig. 2c). When $D=24 \mathrm{~mm}$, the SE rapidly increases from 0.00625 to $0.904 \mathrm{~mJ}$ as $\mu$ amplifies. The increased extent is identified by the slope of the connected segment of two adjacent SE values, which rapidly grows from 0.076 to 0.71 . The exponential growth of SE can be understood by the theoretical relationship between the SE and the thickness of electrodes [30]. We used an IRC in the curved model to illustrate the theoretical analysis (Fig. S8). The SE of the models can be assumed to the SE from $\varepsilon_{11}$, since $\varepsilon_{11}$ is the principal strain. The electrode in the IRC is considered as $n$ of very thin layers with the identical thickness $d l$. For one of them, the SE can be expressed as follows:

$\mathrm{SE}_{\mathrm{TL}}=\frac{E_{\text {electrode }} \times \varepsilon_{i 11}^{2} \times V}{2}$,

where $E_{\text {electrode }}$ and $V$ are the Young's modulus and the volume of the layer, and $\varepsilon_{i 11}$ is the strain in direction 11 in 

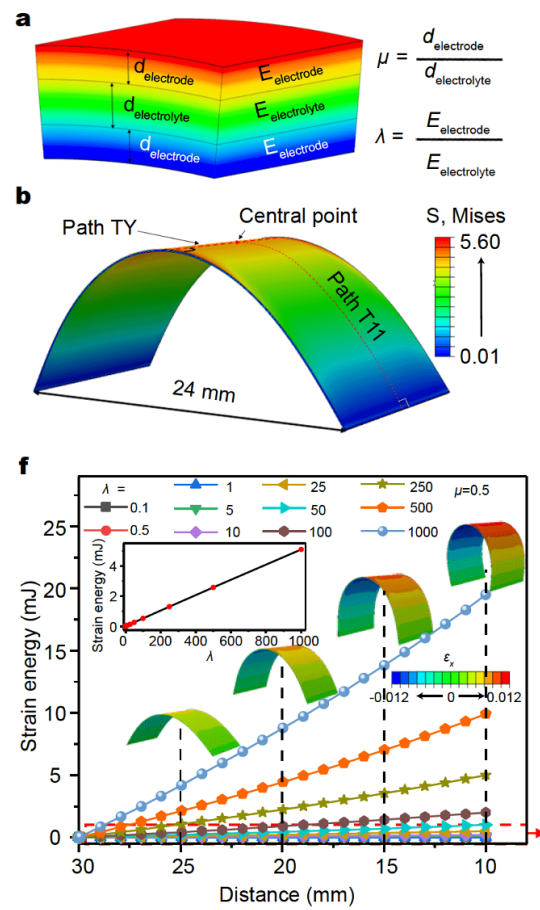
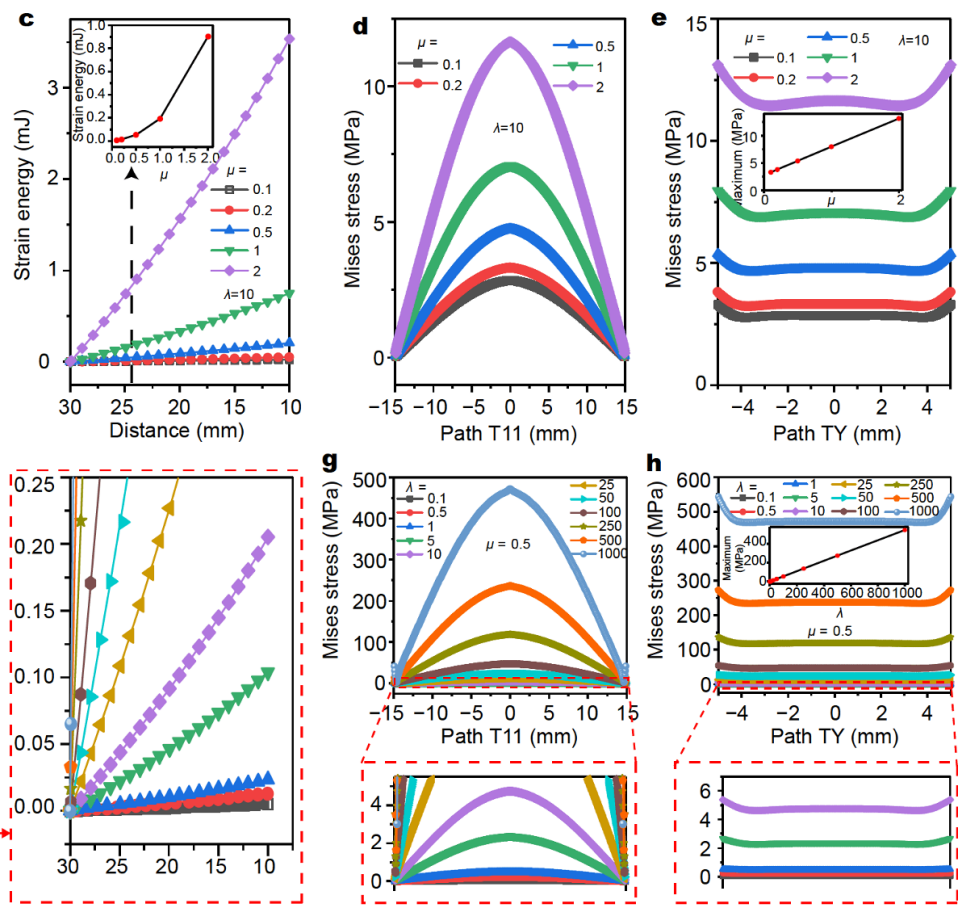

Figure 2 Influences of the thickness and Young's modulus of electrodes on the SE and Mises stress. (a) Definition of the ratio of $d_{\text {electrode }}$ to $d_{\text {electrolyte }}$ and the ratio of $E_{\text {electrode }}$ to $E_{\text {electrolyte }}$ (b) Definition of the paths for obtaining data. (c) Effect of $d_{\text {electrode }}$ on SE. (d) Effect of $d_{\text {electrode }}$ on Mises stress along the Path T11. (e) Effect of $d_{\text {electrode }}$ on Mises stress along Path TY. (f) Effect of $E_{\text {electrode }}$ on SE. (g) Effect of $E_{\text {electrode }}$ on Mises stress along Path T11. (h) Effect of $E_{\text {electrode }}$ on Mises stress along Path TY.

the layer (Fig. S8). According to Equation (1), $\varepsilon_{11}$ can be replaced by $y / \rho$, and therefore

$\mathrm{SE}_{\mathrm{TL}}=\frac{E_{\text {electrode }} \times y_{i}^{2} \times S \times d l}{2 \rho^{2}}$,

where $S$ is the area of the layer surface and $y_{i}$ is the distance between layer $i$ and neutral plane. Accordingly, SE of the electrode in the IRC can be expressed as follows:

$\mathrm{SE}_{\mathrm{IRCE}}=\sum_{i=1}^{n} \frac{2 E_{\text {electrode }} \times S \times d l \times y_{i}^{2}}{2 \rho^{2}}$.

$y_{i}$ can be expressed as $y_{0}+i \times d l$, where $y_{0}$ is the vertical distance between neutral surface and the top or bottom surface of the electrolyte, and therefore

$$
\begin{aligned}
\mathrm{SE}_{\mathrm{IRCE}}= & \frac{E_{\text {electrode }} \times S}{\rho^{2}} \times\left[y_{0}^{2} \times d_{\text {electrode }}\right. \\
& \left.+\sum_{i=1}^{n} 2 y_{0} \times(d l)^{2} \times i+\sum_{i=1}^{n}(d l)^{3} \times i^{2}\right], \\
\mathrm{SE}_{\text {IRCE }}= & \frac{E_{\text {electrode }} \times S}{\rho^{2}} \times\left[y_{0}^{2} \times d_{\text {electrode }}\right. \\
& +y_{0} \times d_{\text {electrode }} \times\left(d_{\text {electrode }}+d l\right)+\frac{1}{6} \times d_{\text {electrode }} \\
& \left.\times\left(d_{\text {electrode }}+d l\right) \times\left(2 d_{\text {electrode }}+d l\right)\right] .
\end{aligned}
$$

The SE of the whole IRC is equal to the SE of electrode and electrolyte, and thus it can be deduced that SE of the whole model could be expressed by a cubic polynomial of the thickness of the electrode. The cubic polynomial regression of the SE values in Fig. 2c can well confirm the deduction (Fig. S9). $R^{2}$ (coefficient of determination) in the regression equates to 1 , which suggests that the cubic polynomial regression equation is almost perfectly fitting all the SE values. The conclusions can be extended to all the bending states. The results indicate that the flexibility of the ESD decreases exponentially with the increase of the thickness of electrodes. Moreover, concerning the two electrodes, the situations of SE are similar to those of the whole model, and their SE accounts for the majority, from $87.4 \%$ to nearly $100 \%$ with the increase of $\mu$ (Figs S10-S12). The results indicate that the increase in the thickness of the electrodes brings about the increase in the SE of the electrodes, which is the main reason for the increase in the SE of the whole ESD. Furthermore, the proportions of the electrode on the top are almost the same to the counterparts on the bottom (Fig. S12). This indicates that the work from tension and compression for the symmetric ESDs is almost the same. This is because 
the tensile modulus and compressive modulus in this study were assumed to be the same.

Mises stress along the four representative paths on the top and bottom surfaces all exhibit intensification with the increase of $\mu$ from 0.1 to 2 (Fig. 2d, e and Figs S5, S7). The maximum stress for each model is obtained, and the slopes of the segments between adjacent values grow with the magnification of $\mu$ in proportion (Fig. 2e). Such results are easy to be comprehended from Equations (1-3), where $\sigma_{11}$ is proportional to $y$ ( $\sigma_{11}$ is the principal stress of Mises stress). The results indicate that the increase in thickness of electrodes gradually raises the possibility of the yield and damage of the electrodes.

With respect to the impact of Young's modulus of electrodes, SE at any bending states increases with the augment of $\lambda$ from 0.1 to 1000 (Fig. 2f). The increased rates are almost the same about 0.005 when $D=24 \mathrm{~mm}$ (the slopes of segments between adjacent SE values). Similar result also can be obtained at other bending states, implying that the flexibility of the ESDs is reduced with the increase of Young's modulus of electrodes. This can be explained by Equation (8), where $\mathrm{SE}_{\mathrm{IRCE}}$ is propor- tional to the Young's modulus of the electrodes. Moreover, similar influences of $E_{\text {electrode }}$ on the electrode components can be obtained, and the proportion of SE of the electrode is increased from $40.0 \%$ to around $98.0 \%$ as $\lambda$ increases from 0.1 to 1000 (Figs S13-S15). The results indicate that the increase in SE of the whole ESD is mainly attributed to the increase in that of the electrodes.

Besides, Mises stress along the four representative paths increases with the magnification of $\lambda$ from 0.1 to 1000 (Fig. 2g, h and Figs S16, S17). The increase of the maximum stress is overall linear, which can be explained by Equations (1-3). The result suggests that the increase in Young's modulus of the electrodes raises the possibility of the yield and damage of the electrodes.

\section{Influences of thickness and Young's modulus of the} electrolyte on strain energy and Mises stress of the ESDs The general situations of SE and Mises stress for each case in Groups 3 and 4 are the same to those for each case in Groups 1 and 2 (Fig. 3a-f and Figs S18-S21).

Regarding the influence of thickness of electrolyte, as $\mu$ diminishes from 2.5 to 0.125 , SE increases at any bending


Figure 3 Influences of the thickness and Young's modulus of the electrolyte on SE and Mises stress. (a) Effect of $d_{\text {electrolyte }}$ on SE. (b) Effect of $d_{\text {electrolyte }}$ on Mises stress along Path T11. (c) Effect of $d_{\text {electrolyte }}$ on Mises stress along the Path TY. (d) Effect of $E_{\text {electrolyte }}$ on SE. (e) Effect of $E_{\text {electrolyte }}$ on Mises stress along Path T11. (f) Effect of $E_{\text {electrolyte }}$ on Mises stress along Path TY. 
states (Fig. 3a). Because $d_{\text {electrolyte }}$ is at the denominator of $\mu$, it is more reasonable to use $1 / \mu$ to identify the change ratio of SE to $d_{\text {electrolyte }}$ SE is increased from 0.13 to $0.5 \mathrm{~mJ}$ with the increase of $1 / \mu$ from 0.4 to 8 . The rapid increase in SE can also be understood by a similar derivation of Equation (8), and the result of the cubic polynomial regression of those SE values, which is $R^{2}=1$, further confirms the deduction (Fig. S22). The analysis suggests that the increase in the thickness of electrolyte also reduces exponentially the flexibility of the ESD. Moreover, the results of SE of the electrodes resemble those of the whole ESD, and the SE proportion of the electrolyte part increases from nearly $0.0 \%$ to $10.0 \%$ with the decrease of $\mu$ (Figs S23-S25).

The results indicate that the increase in the thickness of electrolyte amplifies not only the SE of its own, but also the SE of electrodes. This is attributed to that the SE of the whole IRC is equal to the SE of electrode and electrolyte which widens the distance between the surfaces of electrodes and the neutral plane.

The influences of electrolyte thickness on Mises stress along the four paths are similar to those on SE, which increase as $\mu$ decreases from 2.5 to 0.125 (Fig. 3b, c). The maximum stress gradually increases from 3.168 to 13.594 $\mathrm{MPa}$ as $1 / \mu$ magnifies from 0.4 to 8 (Fig. S26). This can also be explained by Equation (1): the increase in the thickness of electrolyte widens the distance between the surfaces of electrodes and the neutral plane, or in other words widens $y$ in this equation. The results suggest that the increase in the thickness of electrolyte raises the possibility of the yield and damage of electrodes.

Concerning the impact of Young's modulus of electrolyte, SE at any bending states increases with the diminution of $\lambda$ from 100 to 0.2 (Fig. $3 \mathrm{~d}$ ). The increased ratio of the $\mathrm{SE}$ to $1 / \lambda$ is identified, when $D=24 \mathrm{~mm}$, to all about 0.008 (Fig. S27). The results imply that the increase of the Young's modulus of electrolyte reduces the flexibility of the ESDs. In terms of each component, as $\lambda$ diminishes, SE of the electrodes has invisible variations, whilst SE of the electrolyte increases gradually (Figs S28S30). The results indicate that the increase of Young's modulus of electrolyte enhances its own stiffness and accordingly decreases the flexibility of the whole ESD. Different from the situation of SE, Mises stress along the four paths has insignificant variations as $\lambda$ decreases from 100 to 0.2 (Fig. 3e, f). The maximum stress shows minor decrease from 5.43 to $5.17 \mathrm{MPa}$ with the decrease of $\lambda$. This might be because the stiffer electrolyte would slightly constrain the Poisson's ratio effect of electrode. The results indicate that the Young's modulus of electrolyte plays limited roles in stress distributions on the top and bottom surfaces, and in preventing the corresponding electrodes from being damaged.

\section{Poisson's ratio effect of the electrodes and the electrolyte on stress distribution}

According to the results above, it is interesting to identify that the peripheries at the peak of the top surfaces of the curved ESDs are easier to be yielded or damaged. It is probably due to the influence of Poisson's ratio. Fig. 4a shows how this phenomenon occurs. When the ESD model is bent, the electrode on the top is under tension whilst that on the bottom is under compression. Thus, owing to the effect of Poisson's ratio, the top electrode will be under accompanying strains shrinking inward along $y$ axis $\left(\varepsilon_{\mathrm{pt}}\right)$ and becomes narrow, whilst the bottom electrode will be under strains expanding outward along $y$ axis $\left(\varepsilon_{\mathrm{pc}}\right)$ and becomes wide. The different types of displacement in $y$ axis on the top and bottom electrodes give bending moments acting about the 11 axis to the peripheries of the ESD along direction 11. The occurrence of the bending moments renders the peripheries slightly turnup, which elongates the whole lengths of the peripheries, thereby increasing the strains and stresses on the peripheries. The turnup, at the same time, renders the areas near the peripheries slightly concave, which shortens the lengths of the areas in direction 11, thereby decreasing the strains and stresses in the areas. Moreover, $\varepsilon_{11}$ appears higher in the middle of paths along direction 11 , and accordingly the effect of Poisson's ratio is more obvious in the middle of the ESDs.

In order to prove the deduction, the influences of Poisson's ratios of the electrodes and electrolyte (denoted by $v_{\text {electrode }}$ and $v_{\text {electrolyte }}$ ) were investigated. Two groups, namely Groups 5 and 6, were developed (Table S1). Groups 5 and 6 separately include five cases with five different $v_{\text {electrode }}$ and $v_{\text {electrolyte }}$ from 0 to 0.4 , respectively. Mises stress along Path TY of the five models in Group 5 is illustrated in Fig. 4b. The stresses at the ends of the Path TY, as the maximum stress in each case, gradually increase with the increase of $v_{\text {electrode }}$ from 0 to 0.4 (Fig. $4 \mathrm{~b}$ ). When $v_{\text {electrode }}=0$, the stresses along the Path TY are almost constant, which implies a relatively uniform stress distribution. The situations near the ends of the path regarding $U_{z}$ and $\varepsilon_{11}$ are similar to those regarding Mises stress (Fig. $4 c, d$ ), which well confirms the process deduced at the beginning of this section. In addition, the stress distributions along Path TY are not changed with the increase of $v_{\text {electrolyte }}$ (Fig. 4e). This indicates that $v_{\text {electrolyte }}$ has little effect on the stress dis- 

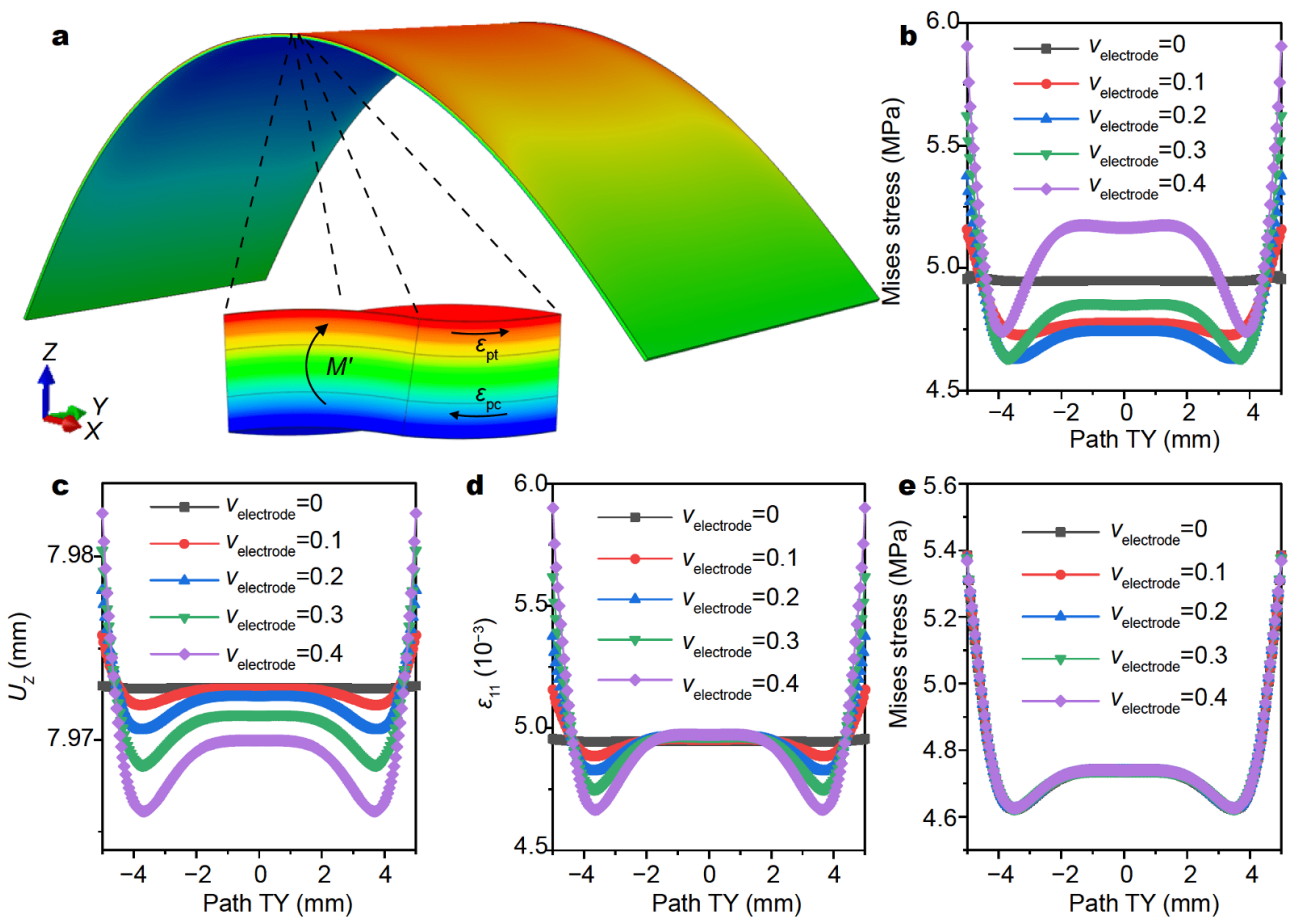

Figure 4 Influences of Poisson's ratios of the electrodes and electrolyte. (a) An illustration of the effect of Poisson's ratio. (b) Effect of $v_{\text {electrode }}$ on Mises stress along Path TY. (c) Effect of $v_{\text {electrode }}$ on displacement along $z$ axis $\left(U_{z}\right)$. (d) Effect of $v_{\text {electrode }}$ on strain in direction $11\left(\varepsilon_{11}\right)$ along Path TY. (e) Effect of $v_{\text {electrolyte }}$ on Mises stress along Path TY.

tributions.

\section{Influences of thicknesses and Young's moduli of the} electrodes and electrolyte on interfacial shear stress When a flexible ESD is bent, shear stresses occur on the interface, which could give rise to the separation between electrodes and electrolyte [6,49]. The interfacial shear stresses still exist in the flexible integrated ESDs, though the whole ESD is integrated [18]. Therefore, it is of paramount significance to investigate the interfacial shear stress for designing the flexible integrated ESDs. The configuration of one integrated ESD model under shear strain is demonstrated in Fig. 5a. According to the definition of direction 11 and $33, \tau_{31}$ on the surfaces of the interfaces can be considered to be interfacial shear stress. In order to obtain more details, four representative paths on the interfaces, namely Path IAN11, Path IANY, Path IBN11 and Path IBNY, were selected (Fig. 5a). Path IAN11 and Path IANY, both above the neutral plane, are along the whole middle line in direction 11 and along the whole edge in direction $y$, respectively. Path IBN11 and Path IBNY are the counterparts of Path IAN11 and Path IANY beneath the neutral plane, respectively. The selection of Path IANY and Path IBNY is based on the numerical work from Yao et al. [50], where the maximum interfacial shear stress was found to be at the two sides of the curved flexible 3D microstructure.

Fig. 5b demonstrates $\tau_{31}$ along Path IAN11 and Path IANY when $\mu=0.5$ and $\lambda=10$ at $D=29,25,20,15$, $10 \mathrm{~mm}$. To make the analysis easy to be understood, we defined $\tau_{31}$ to be positive if it acts in the positive direction 11. The negative sign indicates the opposite direction. The results from Path IBN11 and Path IBNY, and from Path IAN11 and Path IANY correspondingly present overall symmetry about the axis $\tau_{31}=0$ (Fig. S31). Therefore, we only elaborate on the results from Path IAN11 and Path IANY. At each bending state, $\tau_{31}$ along Path IAN11 is overall symmetric about the central point $(0,0)$. From the middle to the ends, $\tau_{31}$ gradually increases and then keeps almost flat. The result confirms that when a flexible integrated ESD is under bending, the interfaces at the two longitudinal ends are easier to be separated. The experimental work from Park et al. [49] also confirmed the conclusion, where when some inorganic filmto-foil silicon electronics began to be bent, the slipping phenomenon, caused by the maximum interfacial shear stress, started from the free edges of the silicon film. Moreover, $\tau_{31}$ along Path IANY is overall symmetric about the axis Path IANY $=0$, and shows gradual decreases from the middle to around $\pm 4 \mathrm{~mm}$ and then sharp 

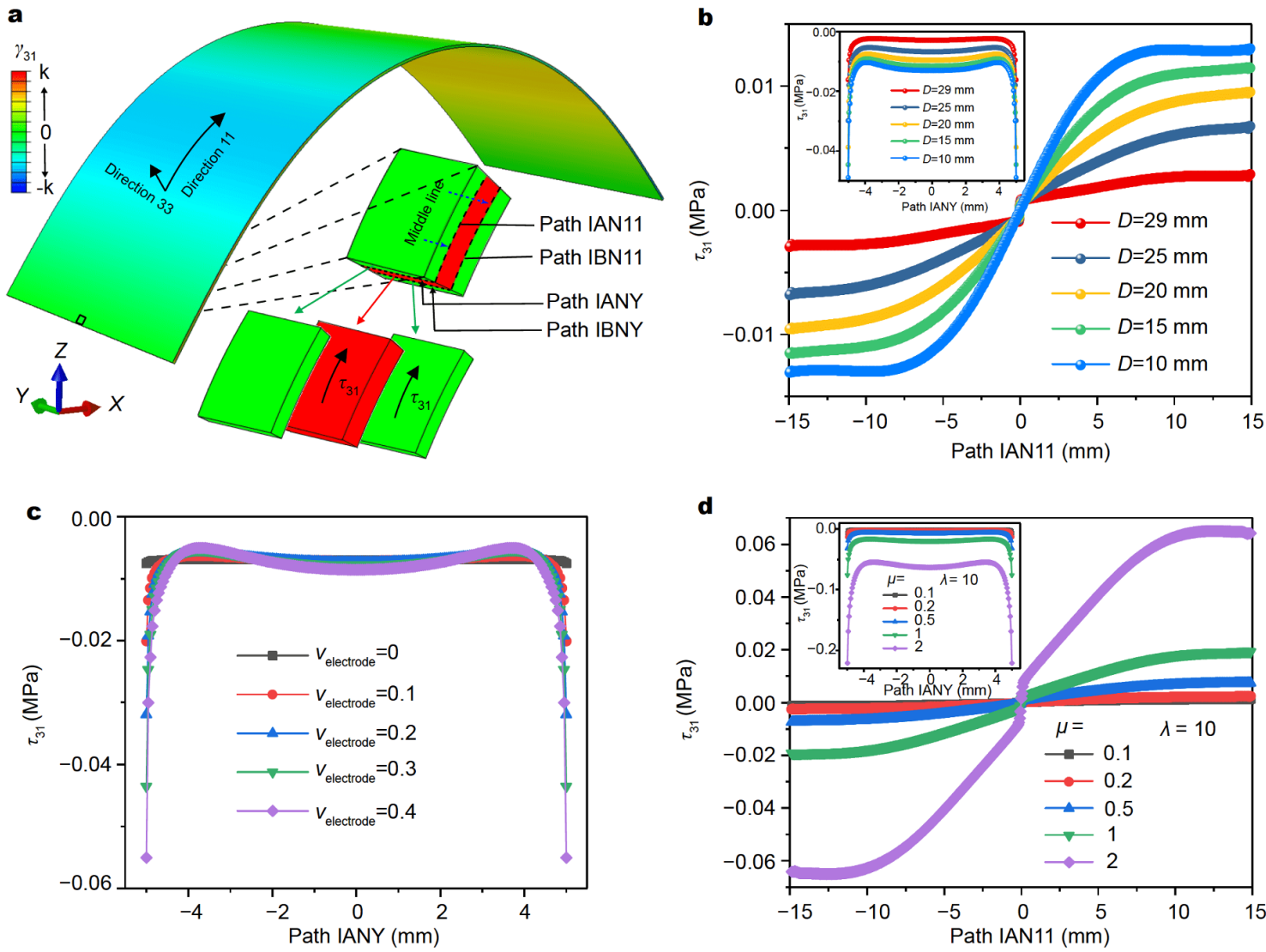

Figure 5 Influences of the thickness and Young's modulus of the electrodes and electrolyte on the interfacial shear stress $\left(\tau_{31}\right)$. (a) An illustration of interfacial shear strain and the definition of paths along the interfaces. (b) Evolution of $\tau_{31}$ along Path IAN11 and Path IANY. (c) Effect of Poission's ratio of electrode ( $v_{\text {electrode }}$ ) on $\tau_{31}$ along Path IANY. (d) Effect of $d_{\text {electrode }}$ on $\tau_{31}$ along Path IAN11.

increases to the ends. The results imply that the four angles of the whole integrated ESD are easier to be separated. The result can also be attributed to the effect of Poisson's ratio of the electrode. The maximum $\tau_{31}$ amplifies with the increase of $v_{\text {electrode }}$, whereas keeps almost constant with the increase of $v_{\text {electrolyte }}$ (Fig. $5 \mathrm{c}$ and Figs S32-S34). In addition, when $D$ decreases from 29 to $10 \mathrm{~mm}, \tau_{31}$ along the four paths is all increased and the maximum $\tau_{31}$ increases from 0.003 to $0.013 \mathrm{MPa}$, which suggests that as the bending extents increase, the ESD is easier to be separated (Fig. 5b and Fig. S31).

The thickness and Young's modulus of the electrodes both have great impact on the interfacial shear stress $\tau_{31}$. When $D=24 \mathrm{~mm}, \tau_{31}$ along the four paths is gradually increased with the increase of $\mu$ from 0.1 to 2 when $\lambda=10$, and also increases with the increase of $\lambda$ from 0.1 to 1000 when $\mu=0.5$ (Fig. $5 \mathrm{~d}$ and Figs S35-S39). The maximum values increase from 0.0073 to $0.22 \mathrm{MPa}$ and from 0.0041 to $1.59 \mathrm{MPa}$, respectively. The results indicate that the thicker and the stiffer the electrodes are, the easier the
ESD is to be separated.

In terms of the electrolyte, both thickness and Young's modulus also influence the shear stress $\tau_{31}$ (Figs S40-S43). When $D=24 \mathrm{~mm}, \tau_{31}$ along the four paths gradually increases as $\mu$ decreases from 2.5 to 0.125 when $\lambda=10$, and also increases as $\lambda$ diminishes from 100 to 0.2 when $\mu$ $=0.5$. The maximum values increase from 0.02 to $0.077 \mathrm{MPa}$ and from 0.019 to $0.13 \mathrm{MPa}$, respectively. The results indicate that the thicker and the stiffer the electrolyte is, the easier the ESD is to be separated.

\section{CONCLUSIONS}

In summary, a systematical analysis was made by the FE method into the mechanical behaviors of the flexible integrated structure symmetric ESDs under bending. It was found that the stiffer and thicker the electrodes are, the harder the integrated ESD is to be bent, and the higher yield strength or failure strain the electrode materials require. The thickness of electrolyte has the similar effect, because it alters the distance between electrodes and the 
neutral plane. In contrast, the influence of Young's modulus of the electrolyte is limited. Furthermore, the peripheries at the peak of the top surface of the bent integrated ESDs, as well as the interfaces at their four angles, were found easier to be damaged due to the effects of Poisson's ratio of electrodes. The higher the Poisson's ratio is, the stronger the effects. Therefore, materials with low Poisson's ratio are recommended to be used for the flexible electrodes. In addition, the thicker and stiffer the electrodes and electrolyte are, the stronger bonding their interfaces require. Although all these conclusions were drawn based on the symmetric integrated ESD models, they would still provide guidance, to some extents, for the design of the unsymmetrical ESDs. This is because the change of the construction would not overturn those pivotal concepts in this work, e.g., the relations based on the fundamental mechanical formula and the reason for the effects of Poisson's ratio. More significantly, this work sheds light on the applications of the FE method in predicting and analyzing the mechanical behaviors of symmetric integrated ESDs during the bending process, which can be extended to systematically studying other types of flexible ESDs under various deformations.

Received 6 November 2020; accepted 8 January 2021; published online 25 March 2021

1 Li Y, Fu J, Zhong C, et al. Recent advances in flexible zinc-based rechargeable batteries. Adv Energy Mater, 2019, 9: 1802605

2 Lin Y, Gao Y, Fan Z. Printable fabrication of nanocoral-structured electrodes for high-performance flexible and planar supercapacitor with artistic design. Adv Mater, 2017, 29: 1701736

3 Xue Z, Song H, Rogers JA, et al. Mechanically-guided structural designs in stretchable inorganic electronics. Adv Mater, 2020, 32: 1902254

4 Li K, Zhang J. Recent advances in flexible supercapacitors based on carbon nanotubes and graphene. Sci China Mater, 2018, 61: 210232

5 Wen L, Li F, Cheng HM. Carbon nanotubes and graphene for flexible electrochemical energy storage: from materials to devices. Adv Mater, 2016, 28: 4306-4337

6 Mao L, Meng Q, Ahmad A, et al. Mechanical analyses and structural design requirements for flexible energy storage devices. Adv Energy Mater, 2017, 7: 1700535

7 Lv T, Liu M, Zhu D, et al. Nanocarbon-based materials for flexible all-solid-state supercapacitors. Adv Mater, 2018, 30: 1705489

8 Yao L, Wu Q, Zhang P, et al. Scalable 2D hierarchical porous carbon nanosheets for flexible supercapacitors with ultrahigh energy density. Adv Mater, 2018, 30: 1706054

9 Li H, Zhang X, Zhao Z, et al. Flexible sodium-ion based energy storage devices: Recent progress and challenges. Energy Storage Mater, 2020, 26: 83-104

10 Xie K, Wei B. Materials and structures for stretchable energy storage and conversion devices. Adv Mater, 2014, 26: 3592-3617

11 Qi D, Liu Z, Liu Y, et al. Suspended wavy graphene microribbons for highly stretchable microsupercapacitors. Adv Mater, 2015, 27:
5559-5566

12 Liu J, Cao H, Jiang B, et al. Newborn 2D materials for flexible energy conversion and storage. Sci China Mater, 2016, 59: 459-474

13 Li H, Tang Z, Liu Z, et al. Evaluating flexibility and wearability of flexible energy storage devices. Joule, 2019, 3: 613-619

14 Simon P, Gogotsi Y. Materials for electrochemical capacitors. Nat Mater, 2008, 7: 845-854

15 Liu W, Song MS, Kong B, et al. Flexible and stretchable energy storage: recent advances and future perspectives. Adv Mater, 2017, 29: 1603436

16 Yao P, Yu J, Zhou J, et al. Engineering 3D electron and ion transport channels by constructing sandwiched holey quaternary metal oxide nanosheets for high-performance flexible energy storage. Sci China Mater, 2020, 63: 1719-1730

17 Yao M, Wang R, Zhao Z, et al. A flexible all-in-one lithium-sulfur battery. ACS Nano, 2018, 12: 12503-12511

18 Yang J, Yu X, Sun X, et al. Polyaniline-decorated supramolecular hydrogel with tough, fatigue-resistant, and self-healable performances for all-in-one flexible supercapacitors. ACS Appl Mater Inter, 2020, 12: 9736-9745

19 Guo T, Zhou D, Liu W, et al. Recent advances in all-in-one flexible supercapacitors. Sci China Mater, 2021, 64: 27-45

20 Wang X, Wang R, Zhao Z, et al. Controllable spatial engineering of flexible all-in-one graphene-based supercapacitors with various architectures. Energy Storage Mater, 2019, 23: 269-276

21 Berger JB, Wadley HNG, McMeeking RM. Mechanical metamaterials at the theoretical limit of isotropic elastic stiffness. Nature, 2017, 543: 533-537

22 Llorca J, González C, Molina-Aldareguía JM, et al. Multiscale modeling of composite materials: a roadmap towards virtual testing. Adv Mater, 2011, 23: 5130-5147

23 Fu H, Nan K, Bai W, et al. Morphable 3D mesostructures and microelectronic devices by multistable buckling mechanics. Nat Mater, 2018, 17: 268-276

24 Liu Q, Lomov SV, Gorbatikh L. When does nanotube grafting on fibers benefit the strength and toughness of composites? Compos Sci Tech, 2020, 188: 107989

25 Arregui-Mena JD, Worth RN, Hall G, et al. A review of finite element method models for nuclear graphite applications. Arch Computat Methods Eng, 2020, 27: 331-350

26 Zhu J, Wierzbicki T, Li W. A review of safety-focused mechanical modeling of commercial lithium-ion batteries. J Power Sources, 2018, 378: 153-168

27 Yuan Z, Wang K, Qiu J, et al. A numerical study on the mechanisms of Dyneema quasi-isotropic woven panels under ballistic impact. Compos Struct, 2020, 236: 111855

28 Zeng H, Yuan Z, Qiu J, et al. Finite element study on the influence of structural parameters on the ballistic performance of $3 \mathrm{D}$ networked fabrics. Appl Compos Mater, 2018, 25: 891-903

29 Koo M, Park KI, Lee SH, et al. Bendable inorganic thin-film battery for fully flexible electronic systems. Nano Lett, 2012, 12: 4810-4816

30 Gere J, Goodno B. Mechanics of Materials, Toronto: Cengage Learning, 2009

31 Li RZ, Peng R, Kihm KD, et al. High-rate in-plane micro-supercapacitors scribed onto photo paper using in situ femtolaserreduced graphene oxide/Au nanoparticle microelectrodes. Energy Environ Sci, 2016, 9: 1458-1467

32 Lin J, Peng Z, Liu Y, et al. Laser-induced porous graphene films from commercial polymers. Nat Commun, 2014, 5: 5714

33 Kumagai S, Mukaiyachi K, Tashima D. Rate and cycle perfor- 
mances of supercapacitors with different electrode thickness using non-aqueous electrolyte. J Energy Storage, 2015, 3: 10-17

34 Tammela P, Olsson H, Strømme M, et al. The influence of electrode and separator thickness on the cell resistance of symmetric cellulose-polypyrrole-based electric energy storage devices. J Power Sources, 2014, 272: 468-475

35 Han M, Wang X, Chen C, et al. All-solid-state supercapacitors with superior compressive strength and volumetric capacitance. Energy Storage Mater, 2018, 13: 119-126

36 Qi L, Song LX, Zhao XF, et al. A facile preparation of flexible alumina/carbon composite nanofibers film. J Nano Res, 2015, 35: $115-127$

37 Yao W, Wang J, Li H, et al. Flexible $\alpha-\mathrm{MnO}_{2}$ paper formed by millimeter-long nanowires for supercapacitor electrodes. J Power Sources, 2014, 247: 824-830

38 Wei J, Wei G, Shang Y, et al. Dissolution-crystallization transition within a polymer hydrogel for a processable ultratough electrolyte. Adv Mater, 2019, 31: 1900248

39 Javaid A, Ho K, Bismarck A, et al. Carbon fibre-reinforced poly (ethylene glycol) diglycidylether based multifunctional structural supercapacitor composites for electrical energy storage applications. J Compos Mater, 2016, 50: 2155-2163

40 Xiong G, He P, Wang D, et al. Hierarchical Ni-Co hydroxide petals on mechanically robust graphene petal foam for high-energy asymmetric supercapacitors. Adv Funct Mater, 2016, 26: 54605470

$41 \mathrm{Yu}$ J, Zhang T, Xu L, et al. Synthesis and characterization of aramid fiber-reinforced polyimide/carbon black composites and their use in a supercapacitor. Chin J Chem, 2017, 35: 1586-1594

42 Cheng J, Chen S, Chen D, et al. Editable asymmetric all-solid-state supercapacitors based on high-strength, flexible, and programmable 2D-metal-organic framework/reduced graphene oxide selfassembled papers. J Mater Chem A, 2018, 6: 20254-20266

43 Di J, Hu D, Chen $\mathrm{H}$, et al. Ultrastrong, foldable, and highly conductive carbon nanotube film. ACS Nano, 2012, 6: 5457-5464

44 Zhang J, Yu Y, Huang D. Good electrical and mechanical properties induced by the multilayer graphene oxide sheets incorporated to amorphous carbon films. Solid State Sci, 2010, 12: 1183-1187

45 Meng C, Liu C, Chen L, et al. Highly flexible and all-solid-state paperlike polymer supercapacitors. Nano Lett, 2010, 10: 4025-4031

46 Wang R, Wang QR, Yao MJ, et al. Flexible ultrathin all-solid-state supercapacitors. Rare Met, 2018, 37: 536-542

47 Shirshova N, Bismarck A, Carreyette S, et al. Structural supercapacitor electrolytes based on bicontinuous ionic liquid-epoxy resin systems. J Mater Chem A, 2013, 1: 15300-15309

48 Javaid A, Zafrullah MB, Khan FH, et al. Improving the multifunctionality of structural supercapacitors by interleaving graphene nanoplatelets between carbon fibers and solid polymer electrolyte. J Compos Mater, 2019, 53: 1401-1409

49 Park SI, Ahn JH, Feng X, et al. Theoretical and experimental studies of bending of inorganic electronic materials on plastic substrates. Adv Funct Mater, 2008, 18: 2673-2684

50 Yao YY, Zhao LP. The bending interface model for flexible threedimensional microstructure. Appl Mech Mater, 2011, 58-60: 10821087

Acknowledgements This work was supported by the National Natural Science Foundation of China (51822205 and 21875121), the Ministry of Science and Technology of China (2019YFA0705600 and
2017YFA0206701), the Natural Science Foundation of Tianjin (18JCJQJC46300 and 19JCZDJC31900), the Ministry of Education of China (B12015) and China Postdoctoral Science Foundation (2019M650045). This work was carried out at the National Supercomputer Center in Tianjin, and the calculations were performed on TianHe-1(A).

Author contributions Yuan $\mathrm{Z}$ developed the FE models, carried out the analyses, and wrote the original draft; Yao $M$ helped to design the numerical experiments and carried out the modelling validation; Zhang $\mathrm{N}$ helped to design the numerical experiments; Wang $\mathrm{S}$ helped to modify the manuscript; Rui X helped to design the experiments; Zhang Q helped to supervise the analysis; Niu Z proposed the concept, provided funding, supervised the modelling analysis and edited the manuscript. All authors contributed to the general discussion.

Conflict of interest The authors declare no conflict of interest.

Supplementary information The details of the model development, verification and validation, and supporting data for the analyses are available in the online version of the paper.

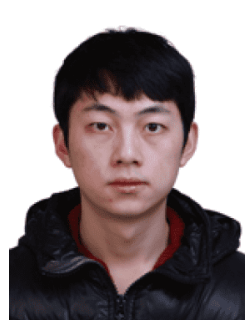

Zishun Yuan received his $\mathrm{PhD}$ in textile science and technology from the University of Manchester, UK (2017). He is currently a postdoctor in the School of Materials and Energy, Guangdong University of Technology. His research covers finite element modelling of flexible energy storage devices.

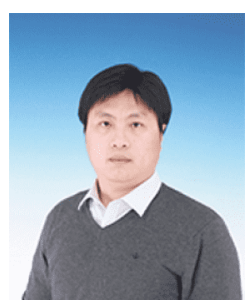

Zhiqiang Niu is currently a Professor at the College of Chemistry, Nankai University. He received his $\mathrm{PhD}$ degree from the Institute of Physics, Chinese Academy of Sciences in 2010. He worked as postdoctor in the School of Materials Science and Engineering, Nanyang Technological University, Singapore (2010-2014). His research interests focus on the unconventional energy storage devices from nanocarbon-based electrode materials to device configurations.

\section{柔性一体化储能器件在弯曲状态下的力学有限元 模拟分析}

袁子舜 ${ }^{1,2}$, 姚敏杰 ${ }^{1}$, 张楠楠 ${ }^{1}$, 王帅 ${ }^{1}$, 茌先宏 ${ }^{2}, 弓$ 其春 $^{3}$, 牛志强 ${ }^{*}$

摘要 近年来, 关于柔性储能器件设计的研究越来越多, 然而对其 在弯曲状态下力学行为的研究还不够系统, 人们对于器件结构所 带来的力学行为影响的认识也不够全面. 在本文中, 我们通过有限 元模拟的方法系统全面地研究了柔性一体化储能器件在弯曲状态 下的力学行为. 本文主要研究了电极和电解质的厚度, 模量以及泊 松比对整个器件在弯曲状态下应变能以及应力应变分散的影响, 所得到的分析结果将为设计拥有更优异力学性能的一体化储能器 件提供指导. 